\title{
Review of: "Tenth year reenrollment randomized trial investigating the effects of childhood probiotics and calcium supplementation on height and weight at adolescence"
}

\section{Lorenzo POLIMENO}

\section{University of Bari}

Potential competing interests: The author(s) declared that no potential competing interests exist.

\section{Dear Editor}

I read the manuscript carefully and to my opinion the data reported does not seem worthy of being published; it reports data already well known in literature (see references 15-19 by the same authors reported). I suggest rejecting the Authors' request to publish the manuscript on Qeios.

Best Regards

Lorenzo Polimeno 